http://jmscr.igmpublication.org/home/

ISSN (e)-2347-176x ISSN (p) 2455-0450

crossref DOI: https://dx.doi.org/10.18535/jmscr/v7i8.31

Journal Of Medical Science And Clinical Research

\title{
Elevated Serum Amylase and Lipase Levels without Actual Pancreatic Involvement in the Presence of DKA- A Cross-Sectional Study
}

\author{
Authors \\ Srividhya $^{1}$, Silambarasan Selvam ${ }^{2}$, Vivek Anand ${ }^{3}$, Ilakkiya Venu ${ }^{4}$ \\ ${ }^{1}$ Assistant Professor, Department of General Medicine, Kanyakumari Government Medical College \\ ${ }^{2,3,4}$ Post Graduate, Department of General Medicine, Kanyakumari Government Medical College
}

\begin{abstract}
Background and Objectives: Diabetic ketoacidosis (DKA) is an acute, major, life threatening complication of diabetes characterised by hyperglycaemia, acidosis, ketonemia/ketonuria. DKA occurs mainly in patients with Type 1 diabetes and also to some extent in patients with Type 2 diabetes. There are case reports with non specific elevation of serum amylase and lipase in DKA patients without actual pancreatic involvement. As the symptoms like nausea, vomiting and abdominal pain can present in both DKA and acute pancreatitis, the elevation of serum amylase and lipase often leads to initial diagnosis of acute pancreatitis. But in the presence of DKA, the patients may need ultrasonogram and CECT abdomen to diagnose acute pancreatitis. So the present study was carried out to study the elevation of serum amylase and lipase levels in patients with DKA and its relevance to the presence of acute pancreatitis.

Materials and Methods: This cross-sectional study was conducted in the Emergency ward of Kanyakumari Government Medical College, Asaripallam during the study period of 6 months from November 2018 to April 2019. A total of 50 patients were included in the study after fulfilling the inclusion and exclusion criteria. A detailed clinical history including history for precipitating factors like omission of insulin and general physical and systemic examination and relevant investigations were done in patients included in the study.

Results: Among the 50 cases studied, 8 cases (16\%) showed elevation in serum amylase levels and 11 cases (22\%) had elevated serum lipase levels. USG abdomen and CECT abdomen were done in patients with elevated enzyme levels, but none of them showed any evidence of acute pancreatitis. This non-specific elevation of lipase was present in more number of cases (22\%) than non-specific elevation of amylase (16\%). So lipase levels elevation appears to be less specific than the amylase levels for the diagnosis of acute pancreatitis.

Conclusion: Elevated levels of serum amylase and lipase can occur in patients with DKA probably due to metabolic derangements and not due to acute pancreatitis.

Keywords: Diabetic ketoacidosis, amylase, lipase.
\end{abstract}

\section{Introduction}

Diabetic ketoacidosis (DKA) remains as serious medical condition causing significant morbidity and mortality, particularly in patients with Type 1 diabetes mellitus and also in patients with Type 2 diabetes mellitus to some extent. DKA is due to relative or absolute deficiency of insulin along with excess of counter regulatory hormones like glucagon, catecholamines, cortisol and growth hormone. The precipitating factors for DKA 
include omission of insulin, infection, acute pancreatitis, acute myocardial infarction and acute cerebrovascular accidents and drugs. The patients may present with nausea, vomiting, polyuria, polydipsia, abdominal pain, generalised weakness and altered mental status. In $50-70 \%$ cases of DKA, abdominal pain may be the presenting symptom mimicking an acute abdomen. In patients with DKA, there is an alteration in various metabolic parameters. The serum amylase and lipase levels in patients with DKA have been studied by various authors but the relationship between the elevated serum amylase and lipase concentration and actual pancreatic involvement is still unclear, probably of non specific elevation.

\section{Objectives}

1. To measure serum amylase and lipase levels in patients with DKA

2. To analyse whether elevated amylase and lipase levels can be present without actual pancreatic involvement in the presence of DKA.

3. To study the correlation between elevated serum amylase and lipase levels and morbidity and mortality of DKA.

\section{Methodology}

\section{Study Design and Study Period}

This is an single centre cross-sectional study including 50 patients admitted in Emergency ward in Kanyakumari Government Medical College during the study period of November 2018 to April 2019.

The patients who fulfilled the criteria for DKA were taken into the study.

\section{Criteria for Diabetic Ketoacidosis:}

1) Hyperglyceamia $\geq 250 \mathrm{mg} / \mathrm{dl}$

2) Metabolic acidosis with $\mathrm{Ph}<7.3$ and/or bicarbonate level $<18 \mathrm{mEq} / \mathrm{L}$

3) Ketonemia/ketonuria

\section{Inclusion Criteria}

1. Age of $>18$ years including males and females

2. DKA of any cause, both type 1 and type 2 DM

\section{Exclusion Criteria}

1. Chronic alcoholics

2. Patients with renal failure

3. Patients with chronic pancreatitis

4. Patients with pancreatic trauma

5. Patients with pancreatic tumours

6. Patients with cholecystitis

7. Patients with intestinal obstruction/bowel perforation

The institutional research and ethical committee clearance was obtained to carry out this study. An informed written consent was obtained from the patients/attenders. A preformatted proforma was used to collect the data. After obtaining a detailed history, general physical and systemic examination, the patients were subjected to relevant investigations.

\section{Observation and Results}

Statistical analysis was done with appropriate statistical tests with $\mathrm{p}<0.05$ as significant. Out of 50 cases, 32 were males(64\%) and 18 were females $(36 \%)$. 8 cases $(16 \%)$ were of type 1 diabetes and 42 cases $(84 \%)$ were of type 2 diabetes. Among the 50 cases, the most frequent precipitating factor was infections seen in 18 cases (36\%), followed by omission of insulin in 12 cases (24\%), acute CVA in 8 cases $(16 \%)$, acute MI in 6 cases (12\%), and unidentified causes in 6 cases $(12 \%)$. 


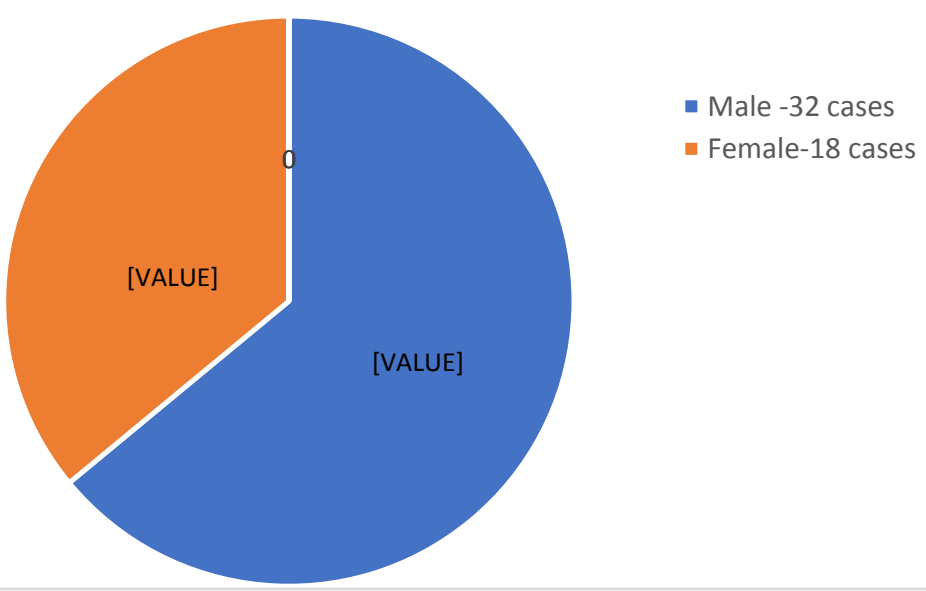

Figure - 1 Distribution of sex in the study

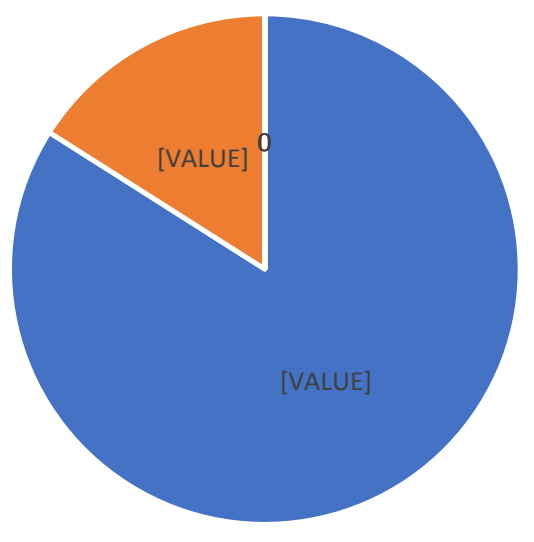

- Type 2 DM-42...

- Type 1 DM-8 cases

Figure - 2 Distribution of Type of DM in the study

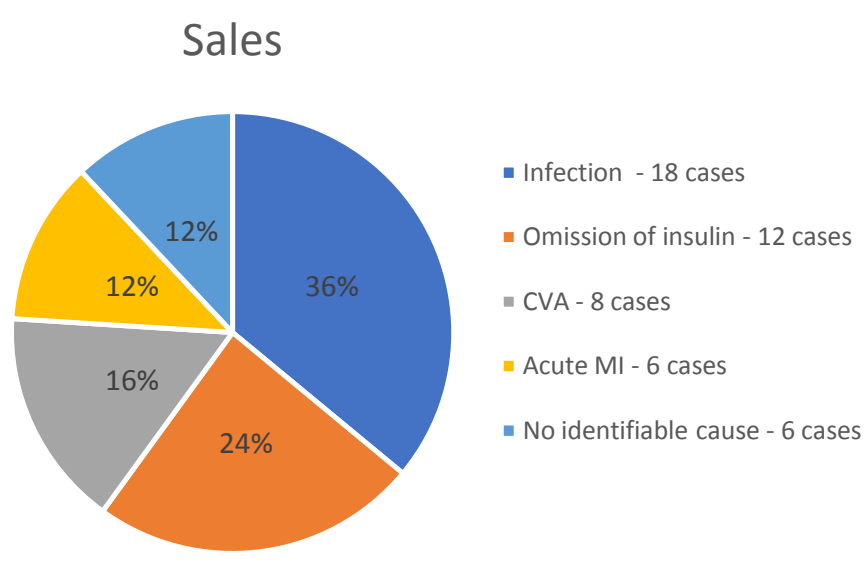

Figure - 3 Distribution of precipitating causes of DKA in this study 
Table 1 Distribution of Amylase, lipase elevation among types of DM

\begin{tabular}{|l|c|c|c|c|}
\hline TYPE OF DIABETES & NORMAL RANGE & LIPASE ELEVATED & $\begin{array}{c}\text { BOTH AMYLASE AND } \\
\text { LIPASE ELEVATED }\end{array}$ & TOTAL \\
\hline Type 1 DM & 5 & 1 & 2 & 8 \\
\hline Type 2 DM & 34 & 2 & 6 & 42 \\
\hline Total & 39 & 3 & 8 & 50 \\
\hline
\end{tabular}

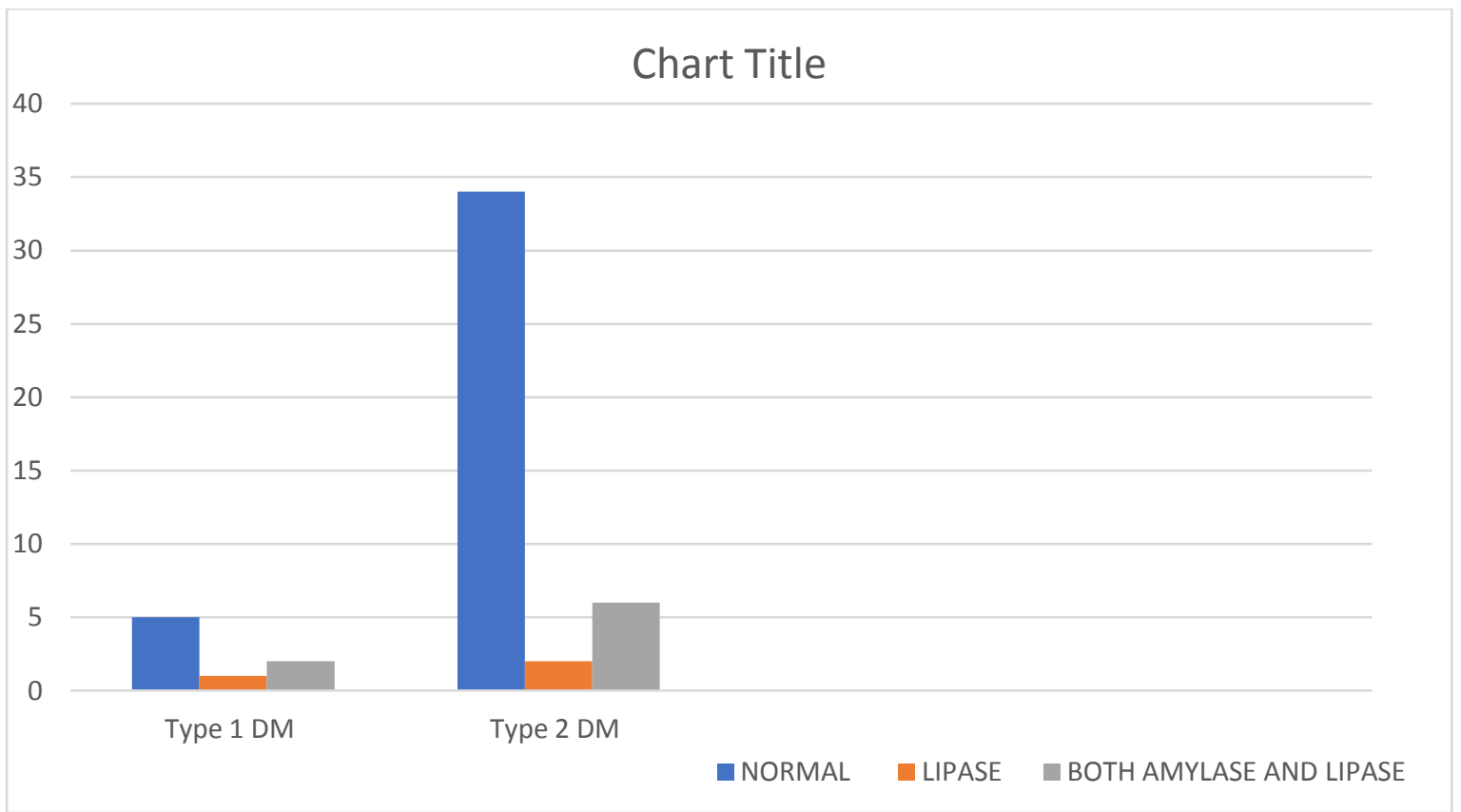

Figure 4 Distribution of serum Amylase, lipase level among types of DM

Among the 50 patients of DKA studied, 8 cases $(16 \%)$ showed elevated serum amylase concentration. They are divided into 2 groups;

1. Group 1: $<3$ times elevation- 3 cases $(6 \%)$

2. Group 2: $\geq 3$ times elevation- 5 cases $(10 \%)$

Number of cases with elevated serum lipase levels was $11(22 \%)$. They are divided into 2 groups:

1. Group 1: $<3$ times elevation- 7 cases $(14 \%)$

2. Group 2: $\geq 3$ times elevation- 4 cases $(8 \%)$ All the cases with elevated levels of serum amylase and lipase, even cases with more than 3 times of elevation were subjected for USG abdomen and CECT abdomen, but none of the cases showed any evidence of acute pancreatitis.

The nonspecific elevation of lipase was present in more number of cases $(22 \%)$ than nonspecific elevation of amylase(16\%). So lipase levels elevation appears to be less specific than elevated amylase levels for the diagnosis of acute pancreatitis. In our study among the 50 cases 3 cases were died, in whom serum amylase and lipase levels were within normal range only. So there is no increased mortality rate among the cases with elevated serum amylase and/or lipase concentration. From the results, there is slightly increased number of cases showing elevated serum levels of amylase and lipase in Type 1 diabetes $(37.5 \%)$ than Type 2 diabetes $(19.04 \%)$.

\section{Discussion}

From various studies it has been identified that in DKA, non specific elevation of serum amylase and lipase levels occurs in 16-25\% of cases. But the exact mechanism for the occurrence of increased enzyme levels without pancreatic involvement is still unclear. The proposed mechanisms include, subtle injury to the pancreatic acinar cells may release enzymes into circulation. Another mechanism is, the metabolic derangements in DKA may trigger the release of enzymes from extra pancreatic tissues like 
amylase from salivary gland and lipase from stomach, liver and small bowel.

\section{Conclusion}

From the present observational study, it is observed that significant, but non specific elevation of serum amylase and lipase levels can be seen in DKA, probably due to metabolic derangements. So increased enzyme levels may also accompany DKA, but not necessarily indicate concomitant pancreatic inflammation. There is no increased mortality attributable to the increased enzyme levels.

\section{Limitations}

The main limitation of this study is small sample size. Further studies with large sample sizes are required.

\section{References}

1. Ali A. Rizvi MD. "Serum amylase and lipase in DKA" Diabetes care November 2003 Vol 26 no.11:3193-3194

2. Yadav D. Nair et al. Nonspecific Hyperamylasemia in DKA: incidence and co-relation with biochemical abnormalities Am J Gastroenterol 2000; 95:3123-8(11)

3. M.C. Vantyghem et al.,(1999) Changes in serum amylase, lipase, leucocyte elastase during DKA and poorly controlled diabetes. Acta Diabetologica. 1999;36(12):39-44.

4. Hameed AM, Lam VWT, Pleass HC. Significant elevations of serum lipase not caused by pancreatitis: a systematic review. HPB (Oxford). 2015;17:99-112

5. Taes YE, Louagie H, Yvergneaux JP, et al. Prolonged hyperlipasemia attributable to a novel type of macrolipase. ClinChem 2000;46:2008-13.

6. Muniraj T, Dang S, Pitchumoni CS. Pancreatitis or not?-Elevated lipase and amylase in ICU patients. J Crit care. 2015;30(6);1370-75. 